\title{
Optimized Feedback-based Traffic Congestion Pricing and Control for Improved Return on Investment (RoI)
}

\author{
Obari Johnson ${ }^{1}$, Sikiru Humble Tajudeen², Muhammed Bashir Mu'azu ${ }^{3}$, Salawudeen Ahmed Tijani ${ }^{4 *}$ \\ 1,2,3 Department of Computer Engineering, Ahmadu Bello University, Zaria, Nigeria \\ ${ }^{4}$ Department of Electrical and Electronics Engineering, University of Jos, Nigeria. \\ Email: ${ }^{1}$ jobari@abu.edu.ng, ${ }^{2}$ thsikiru@abu.edu.ng, ${ }^{3}$ mbmuazu@ abu.edu.ng, ${ }^{4 *}$ atsalawudeen@ unijos.edu.ng \\ *Corresponding Author
}

\begin{abstract}
Traffic congestion is a serious problem in any developing society. One of the approaches used in addressing this problem is congestion pricing. In this paper, the effects of social behavior on congestion pricing and control were considered and a scenario of a $1 \times 2$ traffic tolling system is used. Also, this work considers the rate of return on investment (RoI) of toll facilities in order to justify the worthiness of the design to investors. In earlier works on feedback-based traffic congestion pricing, the traffic parameters in the logit expression were selected arbitrarily and this made it difficult for traffic designers to arrive at optimum parameters within a reasonable amount of time. In order to address this challenge, the traffic parameter problem is formulated into a traffic congestion control optimization problem whose goal is to maximize the congestion price. The constraints are boundaries for the traffic parameters and the investment boundary conditions. The fitness of the formulated optimization problem was determined using genetic algorithm (GA). A number of simulations were performed by considering different multiplication factors and results were obtained for each multiplication factor (m.f). The simulation results justify the exactness of the formulated optimization problem and the superior performance of this work over the one that involves manually selection of traffic parameters.
\end{abstract}

Keywords- RoI, Congestion Pricing, Flat Toll, Genetic Algorithm, Congestion Pricing, Logit Model

\section{INTRODUCTION}

Traffic congestion is one of the major transportation problems in the world. The effects of congestion are imminent in most active settlements in the society [1-3]. Traffic congestion leads to increase in travel times, accidents, disorderliness, and chaos [4-6]. Congestion has posed serious problems by reducing the viable economic activities in major cities or urban centers of the world today $[7,8]$.

The use of both the intelligent and conventional traffic light control has played a significant role in reducing traffic congestion on high ways [9]. However, it is a reasonable approach to turn such congestion problems into like economic benefits. Hence, congestion pricing has been used to address the obstruction to traffic flow on roads [10]. Congestion pricing can be defined as the monetary charging of vehicles for access to certain lanes or roads in order to mitigate traffic congestion at peak hours on major roads by diverting road commuters to alternate lane [11, 12]. Economists' view on road pricing system revealed that for attainment of social equilibrium, the toll is charged at a rate which is equal to the difference between the marginal cost and the average cost for road users [12]. Recent works have revealed the health benefits of congestion pricing; an example is reduction in the rate of asthma attack among children in the city of Stockholm associated with reduction in air pollution due to the introduction of traffic congestion pricing system [13-15]. The development of highly sophisticated sensors has caused advancement in the technology of electronic toll system. Many congestion pricing systems are designed in static condition where the problems are formulated based on traffic data collection $[12,16]$. The advancement in modern technology have made it possible for a dynamic congestion pricing system where the congestion pricing depends on realtime traffic information [17, 18]. A lot of dynamic approaches on the traffic congestion pricing have been proposed in literatures which are mostly based on either experimental design with high complexity or computational design with high cost of computation $[1,2,6]$. The work of Kachroo, Gupta [19] is a state-feedback approach on a 1X2 traffic configuration. The approach gives optimal congestion pricing by linearizing the nonlinear feedback problem using Hamilton Jacobi Bellman's (HJB) equation.

However, the selection of weighting parameters is done arbitrarily by choice or intuition of traffic officers [19-21]. This may not guarantee optimal price in good time as a result of the rigor involved in arriving at the right combination of the traffic parameters. In order to address this problem, this paper formulated Congestion Pricing Optimization Problem (CPOP), with consideration for improved Return on Investment (RoI). We defined the CPOP decision variables as the unknown traffic parameters defined by [12]. We then optimize CPOP using Genetic Algorithm (GA) which is a popular evolutionary for solving various optimization problems [22]. It also has the ability to search solution within an infinite space which makes it suitable for CPOP. In the proposed CPOP model, we used RoI to determine the profit margin of an investment decision or expenditure. It is a key performance indicator that reveals the profitability of a business and also tells the worthiness of investing in our traffic congestion pricing and control scheme. Thus, the proposed model come handy in making the following investment decision: 
1. Making marketing and sales strategy

2. Making purchase of facilities

3. Basis for scalable expansion in tasks and employees' number.

\section{LITERATURE REVIEW}

The problem of congestion on roads has been an interesting problem for a long time. Different contributions have been presented by authors in order to reveal various evolving trends in the field of congestion pricing of roads. Road congestion pricing schemes were simulated using a dynamic equilibrium simulator by examining driver's behavior towards tolling in Palma, Kilani [13, 23]. A logit model was formulated for congestion price and the systemic cost of trip was expressed. Simulations results indicate a better welfare gain on step tolling scheme than flat tolling scheme. However, the assumptions to near equilibrium state with subject of optimality to number of iterations poses uncertainty in this approach. In Lou, Yin [23], optimal congestion pricing scheme was proposed for High Occupancy Toll (HOT) lane. By the use of loop data detector, motorist's willingness to pay was used to obtain a logistic regression model whose parameters are obtained by the use of Kalman filter. Different optimization problems were posed by using the self-lane changing ability of drivers and by partitioning the lanes into cells such that the respective goals of optimization are to maximize sum of the flow rates of the downstream cells and to maximize the congestion price. However, the approaches adopted poses high computation burden. Optimal distance toll for a cordon-based congestion pricing over a continuous distributed value of time was proposed by Meng, Liu [24]. Here, by assuming that time is continuously distributed, a Mixed Integer Programming Problem was imposed on a nonlinear optimal toll distance problem which was solved by hybrid of genetic algorithm and cost averaging method. This work addresses cordon-based congestion pricing, however, the fact that discontinuity unfolds in simplest societal interaction negates the assumption of continuous distributed value of time. Cheng and Han [11] proposed an optimal toll design from the perspective of sustainable development. A framework containing the total vehicular emission, total fuel consumption and total travel time was described in the network. A bi-level optimization problem was established by a sustainable development index (SDI) which is a linear combination of the three stated indicators defined in the framework. Genetic algorithm was used to solve toll design problem at upper level while gradient projection algorithm was used to handle the traffic assignment problem at the lower level [25]. The model is suitable only for a single user group as revealed through implementation on a traffic scenario, however, it cannot handle multi-user group and also did not consider the effect of demand uncertainty. A marginal-cost pricing through trial and error procedure with day-to-day flow dynamics was presented by [22]. The observed link flow pattern was put in disequilibrium by some underlying assumptions which are; the path lows are assumed to trace the 'excess travel cost dynamics' and other assumption on evolution flow dynamics. The update of toll charges is made and consequent convergence of iterative approach to optimality is ensured. There is tradeoff between the cost of implementation and error in this work as there exists a compromise between the number of inter-trial periods and trial number, [26] proposed optimal control for congestion pricing in a dynamic model. A mathematical model for dynamic congestion from the basis of traffic conservation law and greenshield's model was derived by considering a $1 \mathrm{X} 2$ traffic system configuration. Hamilton Jacobi Bellman's equation was used in linearizing the model and establishing the control law. Optimal percent toll was obtained at static condition and this value was used as an input in a logistic regression (logit) expression for congestion price. In order to address the problem with chattering which is inherent in discontinuities, saturation functions were used. This method helps to totally eliminate chattering as compared to the previous work on feedback dynamics of toll system. However, arriving at the optimal value of the congestion price cannot be achieved in an appreciable time because of the rigor involved in the arbitrary selection of traffic weights or parameters by traffic officers in the logit model for congestion price. These parameters are weights on the toll, difference in travel times and driver's choice which need to be optimally selected for proper congestion pricing. Hence, a traffic control optimization problem is formulated whose goal is to maximize the congestion price. The constraints are the investment boundary conditions with the boundaries of the traffic parameters which are the decision variables. Genetic Algorithm is used to obtain the combination of traffic parameters that gives the optimal congestion price.

\section{Methodology}

This section discussed detail methodology adopted to developed the traffic

\section{A. Developing the annuity-based flat toll}

This subsection explains how the flat toll was determined using the concept of annuity. Annuities are series of payments that are made at fixed time intervals over a definite period of time. The frequency of payment made can be monthly, quarterly, semi-annually or annually [27]. Annuities are usually classified as ordinary annuity or due annuity. The focus of this paper is on ordinary annuity with consideration for future value. This future of ordinary annuity is expressed in Eq. (1).

$$
\psi=C \times\left[\frac{(1+i)^{n}}{i}\right]
$$

Where $\Psi=$ Future value of ordinary annuity, $C=$ Cash flow per period, $\mathrm{n}=$ number of payments (concession period in years) and $\mathrm{i}=$ interest rate [12].

In order to formulate a flat toll problem by considering the rate of return on investment, the candidate average congestion price on accumulation with interest is posed to be equal to the amount of investment made on toll facility with respect to the period of concession. Let $\mathrm{P}$ be the total principal used in investment of the road and toll facilities, $r$ to be interest rate in percentage and $\mathrm{A}$ be the total principal amount at the end of $\mathrm{n}$ years. Therefore, $\mathrm{A}$ is expressed as $[12,19,23]$ 


$$
A=P(1+r)^{n}
$$

If the average traffic flow per day of a toll point is ' $t$ ' vehicles/day, and electronic tolling system works for ' $\mathrm{k}$ ' days in a week and then ' $q$ ' weeks in a year. Then, an estimate of the total toll in a year, Y, can be made from Eq. (3)

$$
Y=p_{\text {flat }} \times t \times k \times q
$$

where pf is the flat toll.

The future value of $\mathrm{Y}$ in $\mathrm{n}$ years of investment, assuming in all cases that the interest rate is parallel for both capital investment and RoI, should be equal to the amount in accumulation of the investment capital in $n$ years. This is express as $[12,28]$,

$$
P(1+r)^{n}=p_{\text {flat }} \times t \times k \times q \frac{\left[(1+r)^{n}-1\right]}{r}
$$

Therefore, flat toll can then be computed by Eq. (5),

$$
p_{\text {flat }}=\frac{P(1+r)^{n} \times r}{t \times k \times q\left[(1+r)^{n}-1\right]}
$$

In reality, congestion price at any traffic scenario should at least satisfy the investment return criterion. This will encourage investors, provided the rate of depreciation of toll facilities can be neglected by assuming that maintenance actions are always carried out on the toll facilities while inflation rate is neglected.

In this paper, an example scenario of Lekki admiralty toll gate in Lagos Nigeria, is used. The principal invested on the road is taken as seventy-five billion Naira - $\mathrm{N5B}$, interest rate is $12 \%$ and the concession period is 45 years. Given that toll facility works for 7 days in a week, 52 weeks in a year and that the average number of vehicles/days on the toll lane is 60487 [29]

\section{B. Optimal Congestion Price Formulation}

In [12], a mathematical model for dynamic congestion from the basis of traffic conservation law and greenshield's model was derived. The model was linearized using Hamilton Jacobi Bellman's and a control law was established. Optimal percent tall was then obtained at static conditions to formulate the resulting congestion price $\mathrm{p}(\mathrm{t})$ as logistic regression given by Eq. (6)

$$
p(t)=\frac{1}{a_{2}}\left[\ln \left(\frac{1-\alpha}{\alpha}\right)-a_{1}\left(T_{T}(t)-T_{R}(t)\right)-a_{3}\right]
$$

where, $a_{1}, a_{2}$ and $a_{3}$ are weights that are adjusted to regulate the toll price depending on traffic condition. While a1 is the weight upon the difference in travel times between toll lane and regular lane, $a_{2}$ is the weight on toll rate while $\mathrm{a}_{3}$ is the weight on other factors that are inherent in the choice of the driver, $\alpha, T_{T}(t)$ and $T_{R}(t)$ are percentage toll, total travel time on toll lane, total travel time on regular lane respectively [12].

Rather than reinventing the wheel, we adopt the logistic regression model in Eq. (6) to formulate our congestion price optimization model. Here, we made the traffic parameters a1, $\mathrm{a}_{2}$ and $\mathrm{a}_{3}$ the unknown decision variables. Unlike in [12] where the values of these parameters were arbitrarily assigned, we employed genetic algorithm to optimally determine the right parameter combination that gives the best congestion price. The congestion pricing cost function is formulated as Eq. (7),

$$
C_{\text {price }}(t)=\max \sum_{i=1}^{N} p(t)
$$

S.t:

$a_{1 \min } \leq a_{1} \leq a_{1 \max }$

$a_{2 \min } \leq a_{2} \leq a_{2 \max }$

$$
a_{3 \min } \leq a_{3} \leq a_{3 \max }
$$

where $\mathrm{N}$, is the total number of generated traffic parameters (i.e. Chromosome population). a1 min and a1 max are the lower and upper bound a1 which are experimentally determined, $a_{2 \min }$ and $a_{2 \max }$ are the lower and upper bound of $a_{2}$, also determined through experiment. In order to make a theoretical justification of the driver's choice of behavior which is weighted by $a_{3}$, the cross elasticity of toll is used to define the randomness in traffic behavior. This serves as an input to the decisive choice of driver in accessing either the toll lane or regular lane. However, we assumed that, the cross-sectional effect posed by driver's behavior is made on the flat toll. Thus, the value of $a_{3}$ is determined using Eq. (8):

$$
\left[a_{3 \min }, a_{3 \max }\right]=\left[\eta_{\min }, \eta_{\max }\right] \times p_{f}
$$

where $\eta$ is a measure of the coefficient of cross elasticity of toll defined by $\left[\eta_{\min }, \eta_{\max }\right] \in \eta$.

To cater for the penalty on investment, an addition constrains which penalize the congestion price as a function flat toll are defined as follows:

$$
C_{\text {price }}(t) \geq p_{f}
$$

An investment criterion is that the congestion price at any instant must be greater than or equal to the flat toll i.e the RoI must be greater than or equal to zero. Thus, an extra constraint is imposed on the RoI such that any instantaneous congestion price should return a RoI that is not greater than 1.5, that is:

$$
\frac{C_{\text {price }}(t)-p_{f}}{p_{f}} \leq 1.5
$$

Thus,

The RoI for investment on road/toll facility at any congestion price $C_{\text {price }}(t)$ is expressed by Eq. (11) 


$$
R o I=\frac{C_{\text {price }}(t)-p_{f}}{p_{f}}
$$

Cross elasticity of toll on a lane is used to explain the cross-sectional effects of toll with respect to another lane. This is the degree of responsiveness of toll price on a lane to changes in traffic parameter or external factors on another lane. It can be positive or negative depending on the direction of the cross-sectional effects. In this paper, the cross elasticity of toll on the toll lane with respect to the alternate lane is used to describe random variation in traffic behavior as an input to driver's choice of behavior.

\section{SIMULATION SETUP}

The Genetic algorithm is used to solve the formulated optimization problem. The parameters in Table I shows the defined boundaries for the penalizing traffic parameters $\mathrm{a}_{1}, \mathrm{a}_{2}$ and $a_{3}$. In practice, the effects of drivers' behavior have been taken as negative externality and as such, absolute values of the results of multiplication of $\eta$ with flat toll is used.

\begin{tabular}{lccc}
\multicolumn{4}{c}{ TABLE I. SiMULATION SETUP } \\
\hline \multicolumn{1}{c}{ Traffic Parameters } & Lb & Ub & Remarks \\
\hline $\mathrm{a}_{1}$ & 100 & 1000 & -- \\
$\mathrm{a}_{2}$ & 0.1 & 1.0 & -- \\
$\mathrm{a}_{3}$ & $\eta_{\min } \times p_{f}$ & $\eta_{\max } \times p_{f}$ & $\eta$ \\
\hline
\end{tabular}

$\eta$ : is a random value in the range of $-0.5 \& 0.5, \mathrm{Lb}$ : is Lower bound, $\mathrm{Up}$ : is Upper bound

The Genetic algorithm is used to solve the formulated optimization problem. The parameters in Table 1 shows the defined boundaries for the penalizing traffic parameters $a_{1}, a_{2}$ and $\mathrm{a}_{3}$. In practice, the effects of drivers' behavior have been taken as negative externality and as such, absolute values of the results of multiplication of $\eta$ with flat toll is used. The GA and the input parameters for the Lekki Admiralty tall gate traffic scenarios are defined as follows: Population of chromosome $=100$, Search dimension $=3 ;$ Crossover $=0.7$, Mutation $=0.2$, Iteration $=500$, Traffic-inflow $=2500$ vehicles $/$ hrs, Traffic free flow velocity $=70 \mathrm{~km} /$ hour, maximum traffic density $=80$ vehicles $/ \mathrm{km}$. The multiplication factors of 1.5 and 1.8 are used in simulations which is done with the aid of MATLAB simulation environment.

\section{SIMULATION SETUP}

In this section, the results of simulations are discussed. The flat toll, according to Eq. (5) is computed as 411.30. This is the minimum value a congestion price should have at any instant of operation of toll facility in order to have a good RoI within the defined concession period. The optimal combination of traffic parameters obtained for m.f of 1.5 were obtained as $658.33,0.15$ and -151.03 for $\mathrm{a}_{1}, \mathrm{a}_{2}$ and $\mathrm{a}_{3}$ respectively. This combination of traffic parameters gives the optimal trajectory of the congestion price. Fig. 1 shows the plots of traffic densities on both lanes, the error dynamics, percentage toll and the congestion price trajectory for multiplication factor of 1.5 . From the figure, it can be observed that, the traffic density of the toll lane begins to reduce at the operation of the toll facility. This is due to social behavior of the commuters as seen in Fig 1a. It reduces until it gets to a point where allowable user equilibrium is attained. On the other hand, in Fig 1b, the traffic density on the regular lane increases till it attains the equilibrium point where drivers of the same source and destination will have the same minimum travel time. The error reduces till it gets to zero at the point of allowable user-equilibrium as seen in Fig 1c. The trajectory of the percentage toll exhibits similar behavior like the traffic density in Fig 1d. In Fig. 1e, the trajectory of the congestion price indicates the satisfaction of investment criterion at every point on the curve till it reaches infinity. This point at infinity indicates the justifiable economic position for congestion price at the allowable userequilibrium. For $m . f$ of 1.8 , the best combination of traffic parameters $a_{1}, a_{2}$ and $a_{3}$ at optimal point after a few runs of simulation were obtained to be $559.72,0.14$ and -134.77 respectively. Fig. 2 shows the plots of traffic densities on both lanes, error dynamics, percentage toll and the trajectory of the congestion price for $m . f$ of 1.8. From Fig. 2, it can also be observed that the traffic density of toll lane at $1.8 \mathrm{~m} . f$ behaves almost the same way as that of $m . f$ of 1.5 , as seen in Fig 2 a. However, it did rise after the initial fall before arriving at the user equilibrium when the error reaches zero as seen in Fig 2c. The percentage toll in Fig. 2 d exhibits similar behavior at this value of $m . f$ like the traffic density of toll lane. The traffic density of the regular lane rises faster and higher than that of $m . f$ of 1.5 which is due to significant difference in travel times posed by changes in $m . f$ before it stabilized or reached the allowable user-equilibrium as seen in Fig $2 \mathrm{~b}$. The trajectory of the congestion price in Fig 2e reveals how it satisfies the investment criteria.

Fig. 3 shows the trajectories of congestion price for both $m . f s 1.5$ and 1.8 for manually selected traffic parameters. For $1.5 \mathrm{~m} . f$, values of $658.33,0.15$ and -130 are chosen as respective values of a1, a 2 and a3. For $m . f$ of 1.8 , the selected values of $\mathrm{a} 1, \mathrm{a} 2$ and $\mathrm{a} 3$ are 559.72, 0.14, and -120 respectively. These manually selected points are close to the points of optimality for both $m$.fs. It can be observed from Fig $3 \mathrm{a}$ and Fig $3 \mathrm{~b}$ that both trajectories satisfy the investment criteria. 


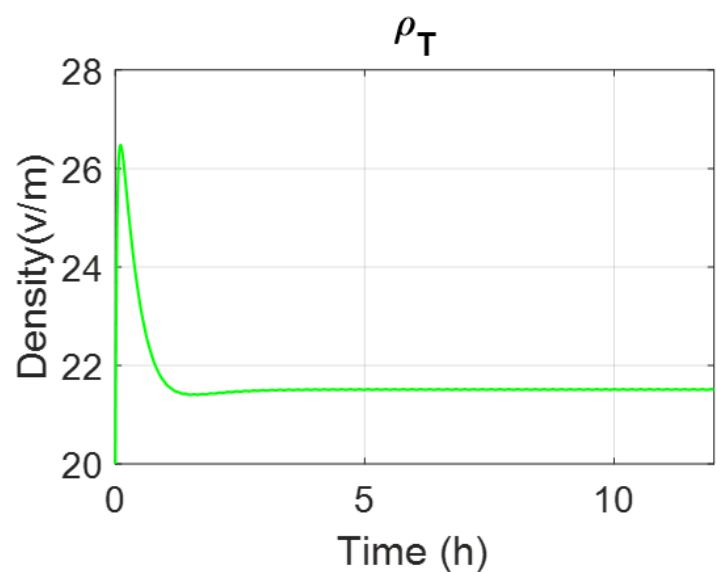

a. Traffic density of toll lane

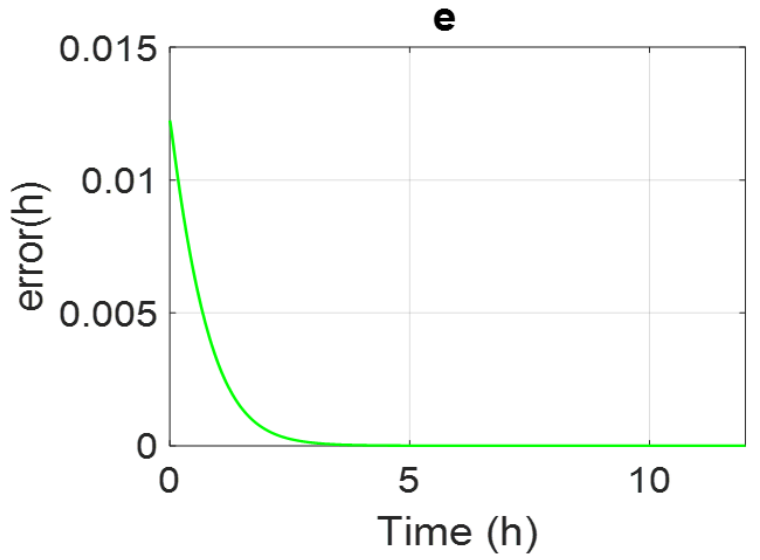

c. Percentage toll

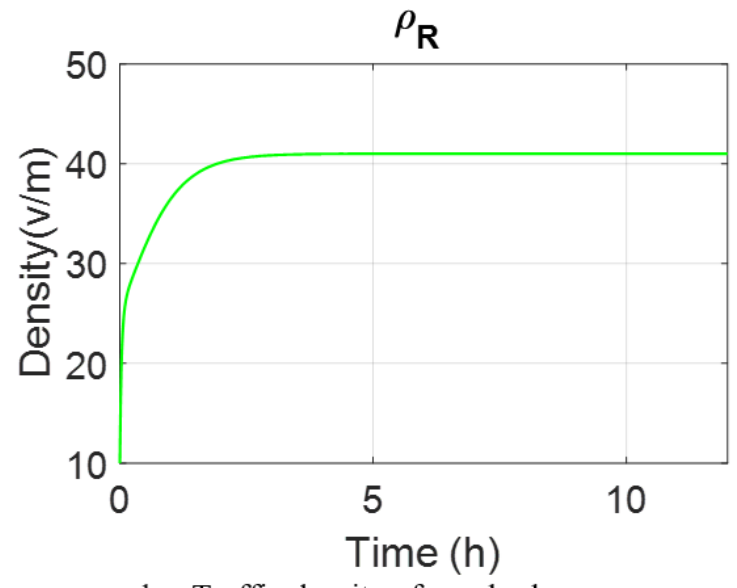

b. Traffic density of regular lane

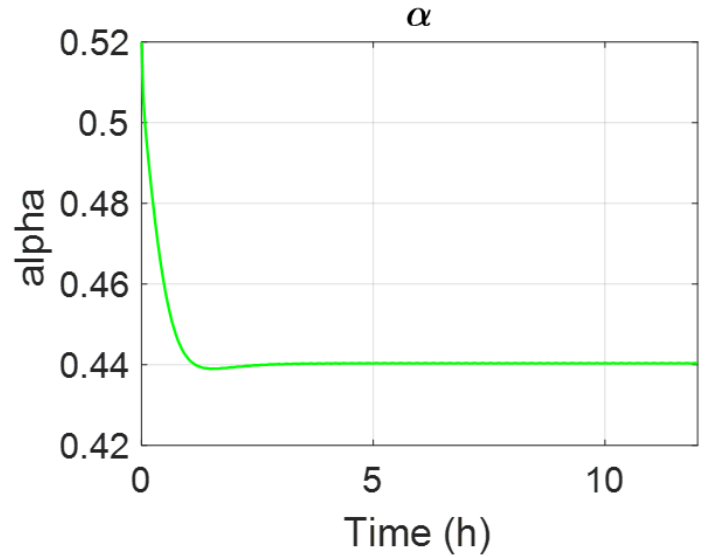

d. Error

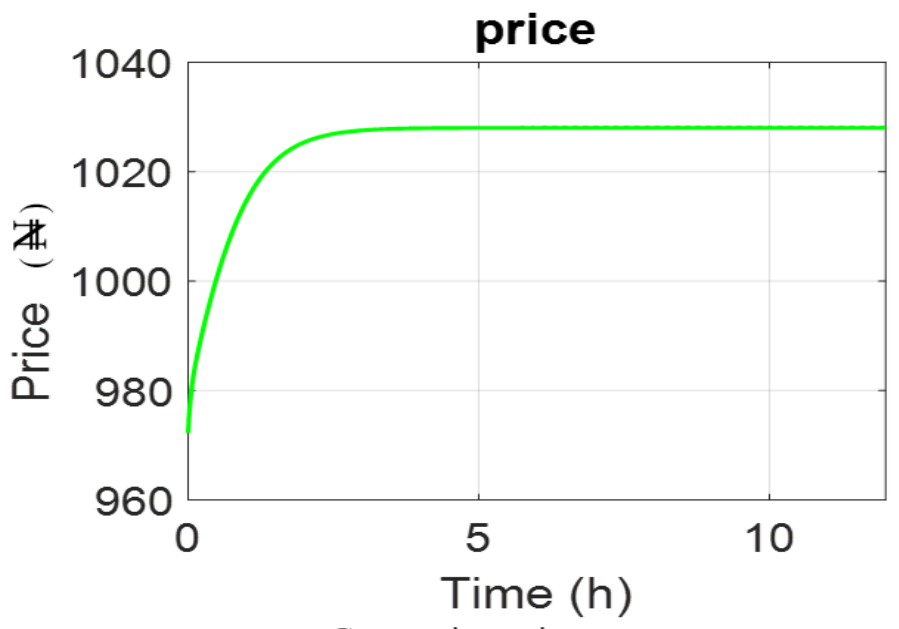

e. Congestion price

Fig. 1. Traffic dynamics and Congestion price for $\mathrm{m} . \mathrm{f}=1.5$

The congestion price increases until the equilibrium is reached. The process of making arbitrary selection of traffic parameters may lead to optimality but with much rigor and computational requirements. The RoI which is used as performance metric was computed at different operation time of toll facility using Eq. (7). Table 2 and Table 3 show the different values of RoI for $m . f s$ of 1.5 and 1.8 . 


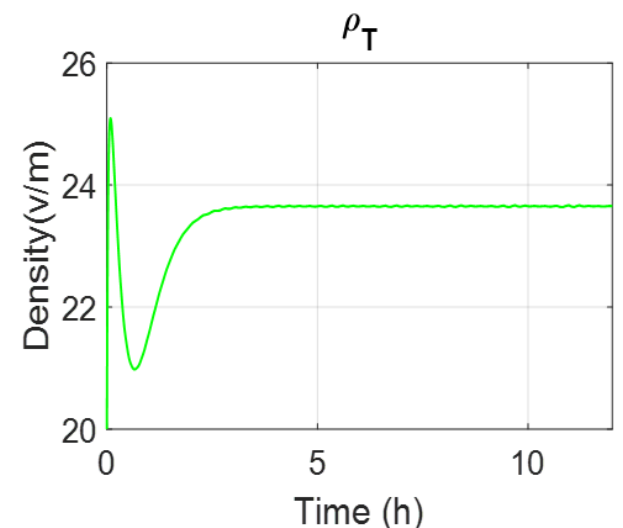

a. Traffic densities of toll lane

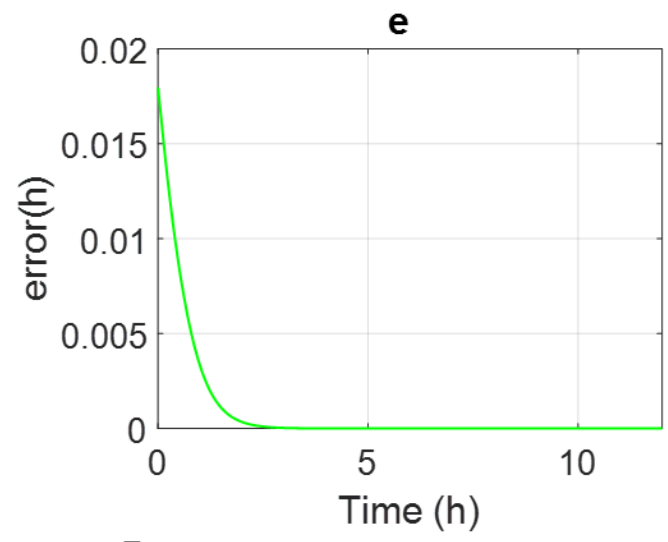

c. Error

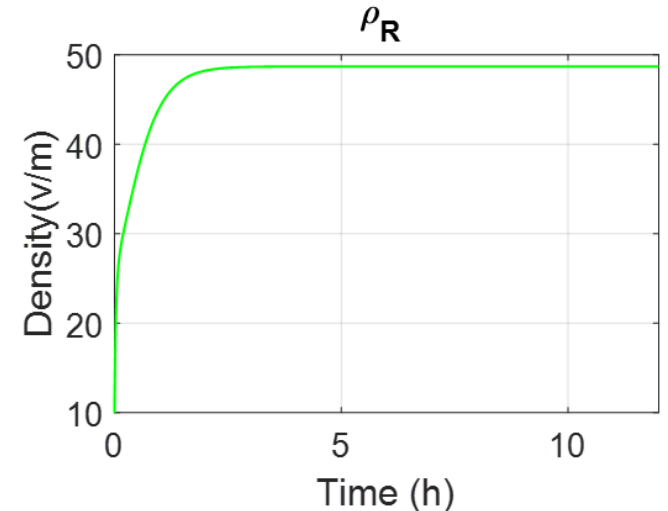

b. Traffic densities of regular lane

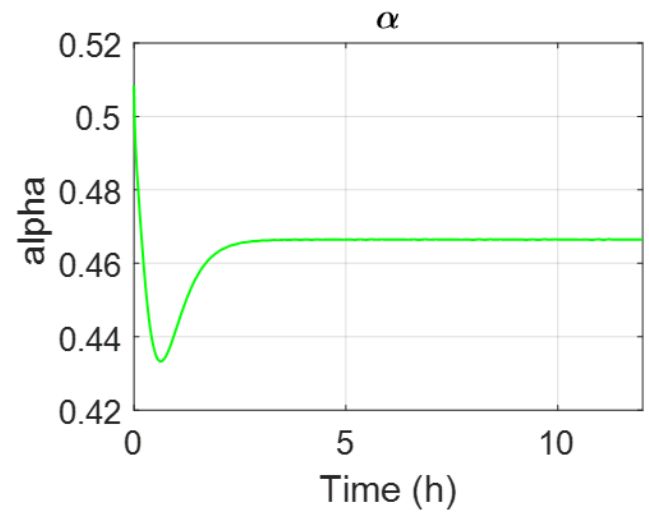

d. Percentage toll

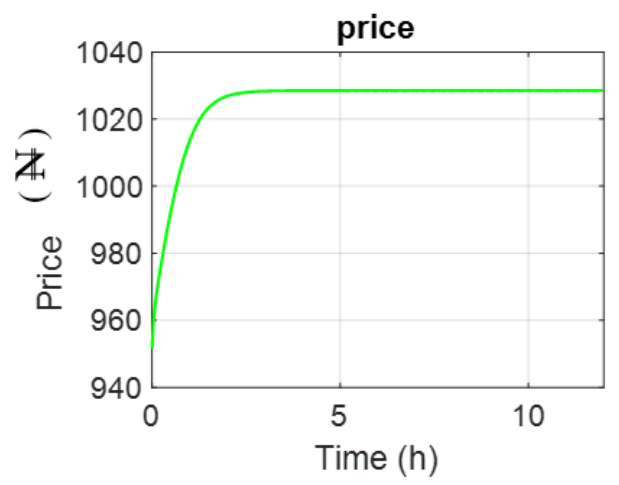

e. Congestion price

Figure 2. Traffic dynamics and congestion price for $\mathrm{m} . \mathrm{f}=1.8$

TABLE II ROI FOR MULTIPLICATION FACTOR OF 1.5

\begin{tabular}{ccc}
\hline Operation time (hrs) & $\begin{array}{c}\text { RoI for arbitrary } \\
\text { selection of traffic } \\
\text { parameters }\end{array}$ & $\begin{array}{c}\text { RoI for optimally } \\
\text { selected traffic } \\
\text { parameters }\end{array}$ \\
\hline 1 & 1.130 & 1.468 \\
2 & 1.160 & 1.492 \\
3 & 1.164 & 1.500 \\
4 & 1.164 & 1.500 \\
\hline
\end{tabular}

TABLE III ROI FOR MULTIPLICATION FACTOR OF 1.8

\begin{tabular}{ccc}
\hline $\begin{array}{c}\text { Operation time }(\mathrm{t}) \\
\text { hours }\end{array}$ & $\begin{array}{c}\text { RoI for arbitrary } \\
\text { selection of traffic } \\
\text { parameters }\end{array}$ & $\begin{array}{c}\text { RoI for optimally } \\
\text { selected traffic } \\
\text { parameters }\end{array}$ \\
\hline 1 & 1.210 & 1.465 \\
2 & 1.241 & 1.497 \\
3 & 1.244 & 1.500 \\
5 & 1.244 & 1.500 \\
\hline
\end{tabular}

Table 3 and Table 4 show that the RoI for the optimally selected traffic parameters is higher than the RoI for manually selected traffic parameters. It can be observed that RoI increases from operating $\mathrm{t}=1 \mathrm{hr}$ to $\mathrm{t}=3 \mathrm{hr}$ where it retains its value for the rest of the period because of the equilibrium attainment. Investment on road and toll designs by investors need to be proven by checking the investment worthiness; RoI is a good indicator of business worthiness.

The result of this work has justified that RoI is improved by formulating traffic congestion pricing optimization problem. Hence, this work justifies the need of investing in road congestion management.

\section{CONCLUSION}

This research presents an optimized feedback-based traffic congestion pricing system and control for improved 
return on investment (RoI). It also provides a detailed extension to the work of Kachroo, Gupta [12] where the traffic parameters in the derived logit expression of congestion price were selected manually. The principal uniqueness of this research work is the development of congestion pricing system and control with consideration of RoI. In order to achieved improved RoI on road and toll design, an investment boundary condition was established by formulation of an expression for flat toll. An optimization problem is formulated with the goal of maximizing the congestion price whose constraints are the boundaries imposed on traffic parameters and the investment boundary conditions. The investment conditions are established by formulating an annuity-based flat toll. Genetic Algorithm (GA) is used to obtain the best combination of traffic parameters that yield optimal trajectory for the congestion price at respective m.fs of 1.5 and 1.8. Also, manual selection of traffic parameters at a point near the optimal solution was done in order to validate the research work at respective m.f and RoI is used as the performance metric.

\section{ACKNOWLEDGMENT}

All the authors wish to acknowledge the insightful contribution of the control and computer research group of the department of computer engineering, Ahmadu bello University, Zaria Nigeria.

\section{REFERENCES}

[1] Y. Long, "Pricing and congestion management based on improved chaos genetic algorithms," in 2016 Chinese Control and Decision Conference (CCDC), 2016, pp. 531-535.

[2] Q. Luo, "Dynamic Congestion Pricing for Ridesourcing Traffic: a Simulation Optimization Approach," in 2019 Winter Simulation Conference (WSC), 2019, pp. 2868-2869.

[3] Z. Nannan, J. Yuanwei, and Z. Siying, "Pricing and congestion management with different users' utility functions in networks," in 2008 IEEE International Conference on Automation and Logistics, 2008, pp. 2994-2998.

[4] S. N. Pandey, S. Tapaswi, and L. Srivastava, "Nodal congestion price estimation in spot power market using artificial neural network," IET Generation, Transmission \& Distribution, vol. 2, no. 2, pp. 280-290, 2008.

[5] Q. Tian and G. Wang, "Congestion Pricing in Two-airport System with Commercial Operations," in 2011 Fourth International Joint Conference on Computational Sciences and Optimization, 2011, pp. 1087-1090.

[6] Y. Yuanzhou, C. Shaokuan, H. Rong, L. Xiao, and M. Baohua, "Optimal road congestion pricing based on game theory," in 2010 Chinese Control and Decision Conference, 2010, pp. 4186-4189.

[7] A. Atta, S. Abbas, M. A. Khan, G. Ahmed, and U. Farooq, "An adaptive approach: Smart traffic congestion control system," Journal of King Saud University - Computer and Information Sciences, 2018/10/23/2018.

[8] C. Onyeneke, C. Eguzouwa, C. J. B. S. Mutabazi, and Informatics, "Modeling the Effects of Traffic Congestion on Economic ActivitiesAccidents, Fatalities and Casualties," vol. 3, no. 2, pp. 7-14, 2018.
[9] A. Brousselle, T. Benmarhnia, and L. J. P. m. r. Benhadj, "What are the benefits and risks of using return on investment to defend public health programs?," vol. 3, pp. 135-138, 2016.

[10] D. Chen, J. Ignatius, D. Sun, M. Goh, and S. Zhan, "Impact of congestion pricing schemes on emissions and temporal shift of freight transport," Transportation Research Part E: Logistics and Transportation Review, vol. 118, pp. 77-105, 2018/10/01/ 2018.

[11] L. Cheng, F. J. D. D. i. N. Han, and Society, "Optimal road toll design from the perspective of sustainable development," vol. 2014, 2014.

[12] P. Kachroo, S. Gupta, S. Agarwal, and K. J. I. T. o. I. T. S. Ozbay, "Optimal control for congestion pricing: Theory, simulation, and evaluation," vol. 18, no. 5, pp. 1234-1240, 2016.

[13] A. De Palma, M. Kilani, R. J. T. R. P. A. P. Lindsey, and Practice, "Congestion pricing on a road network: A study using the dynamic equilibrium simulator METROPOLIS," vol. 39, no. 7-9, pp. 588-611, 2005.

[14] H. Gehlot, H. Honnappa, and S. V. Ukkusuri, "An optimal control approach to day-to-day congestion pricing for stochastic transportation networks," Computers \& Operations Research, vol. 119, p. 104929, 2020/07/01/ 2020.

[15] Z. Gu, Z. Liu, Q. Cheng, and M. Saberi, "Congestion pricing practices and public acceptance: A review of evidence," Case Studies on Transport Policy, vol. 6, no. 1, pp. 94-101, 2018/03/01/ 2018.

[16] F. Harrou, A. Zeroual, and Y. Sun, "Traffic congestion monitoring using an improved kNN strategy," Measurement, vol. 156, p. 107534, 2020/05/01/ 2020.

[17] M. B. Kadri and W. A. J. T. S. W. J. Khan, "Application of genetic algorithms in nonlinear heat conduction problems," vol. 2014, 2014.

[18] Y. Lou, Y. Yin, and J. A. J. T. R. P. C. E. T. Laval, "Optimal dynamic pricing strategies for high-occupancy/toll lanes," vol. 19, no. 1, pp. 64-74, 2011.

[19] Q. Meng, Z. Liu, S. J. T. R. P. E. L. Wang, and T. Review, "Optimal distance tolls under congestion pricing and continuously distributed value of time," vol. 48, no. 5, pp. 937-957, 2012.

[20] T. Seo, "Trial-and-Error Congestion Pricing Scheme for Morning Commute Problem with Day-to-Day Dynamics," Transportation Research Procedia, vol. 47, pp. 561-568, 2020/01/01/ 2020.

[21] E. Simeonova, J. Currie, P. Nilsson, and R. Walker, "Congestion pricing, air pollution and children's health," National Bureau of Economic Research0898-2937, 2018.

[22] H. Ye, H. Yang, and Z. J. T. R. P. B. M. Tan, "Learning marginal-cost pricing via a trial-and-error procedure with day-to-day flow dynamics," vol. 81, pp. 794-807, 2015.

[23] A. de Palma and R. J. T. R. P. C. E. T. Lindsey, "Traffic congestion pricing methodologies and technologies," vol. 19, no. 6, pp. 13771399, 2011.

[24] Q. Zhao, S. Chen, S. Leung, K. J. T. R. P. E. Lai, Logistics, and T. Review, "Transportation Research Part E, Logistics and Transportation Review," vol. 46, pp. 913-925, 2010.

[25] E. T. Verhoef, "Optimal congestion pricing with diverging long-run and short-run scheduling preferences," Transportation Research Part B: Methodological, vol. 134, pp. 191-209, 2020/04/01/ 2020.

[26] B.-W. Wie and R. L. J. T. R. P. B. M. Tobin, "Dynamic congestion pricing models for general traffic networks," vol. 32, no. 5, pp. 313327, 1998.

[27] D. Metz, "Tackling urban traffic congestion: The experience of London, Stockholm and Singapore," Case Studies on Transport Policy, vol. 6, no. 4, pp. 494-498, 2018/12/01/ 2018.

[28] C. P. Chu, C. Y. Wang, and S. R. Hu, "Application of E-Tag in Pricing Road Tolls and Parking Fees for Traffic Congestion Mitigation," Transportation Research Procedia, vol. 25, pp. 2913-2922, 2017/01/01/2017.

[29] O. Oluwafemi, "Lagos State Explains it Saved Motorist N87 Billion Annually by Removing Lekki Roundabouts," Lagos State Journalist, 2017. 